DESPRET Vinciane, 2019, Habiter en oiseau, Actes Sud (coll. Mondes sauvages), Arles, 208 p.

\title{
Mélanie Bourlet
}

\section{OpenEdition}

\section{Journals}

Édition électronique

URL : https://journals.openedition.org/clo/8755

DOI : $10.4000 /$ clo.8755

ISSN : 2266-1816

Éditeur

INALCO

\section{Édition imprimée}

Date de publication : 30 juin 2020

Pagination : 239-243

ISBN : 978-2-85831-392-1

ISSN : 0396-891X

\section{Référence électronique}

Mélanie Bourlet, « DEsPRET Vinciane, 2019, Habiter en oiseau, Actes Sud (coll. Mondes sauvages), Arles, 208 p. ». Cahiers de littérature orale [En ligne], 87 | 2020, mis en ligne le 16 septembre 2021, consulté le 09 novembre 2022. URL : http://journals.openedition.org/clo/8755 ; DOI : https://doi.org/10.4000/clo. 8755

\section{(c) (i) (2)}

Creative Commons - Attribution - Pas d'Utilisation Commerciale 4.0 International - CC BY-NC 4.0 https://creativecommons.org/licenses/by-nc/4.0/ 


\title{
DESPRET Vinciane, 2019, Habiter en oiseau, Actes Sud (coll. Mondes sauvages), Arles, 208 p.
}

\author{
Mélanie Bourlet \\ Inalco, Llacan (UMR8135)
}

Voilà bien un drôle d'oiseau que ce petit livre de la philosophe belge Vinciane Despret, dédié à Donna Haraway, Bruno Latour et Isabelle Stengers et postfacé par Baptiste Morizot et Stéphane Durand, et qui nous invite à penser avec les ornithologues ce que pourrait signifier « habiter » du point de vue d'un oiseau. Pour ce faire, l'autrice opère par une série de déplacements progressifs destinés à ouvrir l'imagination.

Et cela commence d'abord par une émotion : celle de Vinciane Despret qui se laisse affecter par le chant d'un merle, si intense qu'il déclenche chez elle la volonté de comprendre ce qui se joue dans cette manière de chanter à tue-tête au sortir de l'hiver avec tout « l'enthousiasme de son corps ». «Quelque chose importe, plus que tout, et plus rien d'autre n'importe si ce n'est le fait de chanter $[\ldots] \gg$ (p. 14). Saisie par cette importance, subjuguée par ce qu'elle qualifie de « roman radiophonique » (p. 13), Vinciane Despret entreprend alors une enquête, dans la littérature ornithologique du $\mathrm{xx}^{\mathrm{e}}$ siècle, sur le sens de cette métamorphose extravagante et exubérante (dans les chants, les couleurs, les mouvements, les postures agressives) au moment où les oiseaux construisent leurs territoires.

C'est donc à une histoire des idées sur le territoire des oiseaux prises dans un jeu permanent d'allers-retours, d'hésitations, d'intuitions, de reprises et parfois d'abandons que Vinciane Despret nous convie. Une investigation rigoureuse, concise et précise, menée tambours battants mais qui se veut aussi un « éloge de la lenteur » (p. 200), selon les mots de Stéphane Durand : l'éloge des savoirs 
patiemment cumulés dans l'observation par les ornithologues, les éthologues, les biologistes et les écologues, pour lesquels la philosophe ressent une profonde admiration. Ce contenu foisonnant est présenté au prisme des petites anecdotes, des expérimentations et des trajectoires de vie des oiseaux, donnant à ce livre un rythme particulier, plein de vivacité. Car la joie est chez Vinciane Despret, une condition importante de la connaissance. Soulignons ici que la structure de ce petit ouvrage n'est pas étrangère à cet effet rythmique. Composée de deux accords et de huit contrepoints, elle se veut polyphonique et musicale, laissant entendre toujours plus de points de vue, comme autant de lignes mélodiques parallèles. Désireuse d'éviter l'écueil anthropocentriste (en projetant sur les oiseaux des qualités humaines ou en tirant de l'observation des oiseaux des leçons pour les humains), Vinciane Despret embarque donc le lecteur dans un exercice de pensée critique virevoltant et accessible, exemplaire par sa méthode et la manière dont il convoque les théories et références de tous bords (philosophiques, ornithologiques, économiques, littéraires, etc.) et crée un mode d'attention entièrement tourné vers les oiseaux et celles/ceux qui les observent. Il importe en effet à la chercheuse de pouvoir « honorer leur existence » (p. 38).

Le premier accord (composé des trois premiers chapitres : «Territoires », « Les Puissances d'affecter », « Surpeupler ») s'attache ainsi à en poser les conditions préalables, en rappelant combien le mot «territoire » a été associé pour les êtres humains au sens de «propriété exclusive » dès le XVII ${ }^{\mathrm{e}}$ siècle, avec pour triste conséquence l'éradication des communs. « Le "territoire" est un terme qui n'a rien d'innocent et dont je ne dois pas oublier les violences appropriatives et les destructions qui ont configuré certaines de ses significations actuelles » (p. 26). Elle rappelle notamment que c'est au $\mathrm{XVII}^{\mathrm{e}}$ siècle que l'attention s'est focalisée sur les oiseaux chanteurs, capturés pour être mis en cage, et qu' « aimer » les oiseaux a pu signifier aussi les tuer pour les collectionner, se les approprier et les étudier. Dans cette perspective, qui sera renforcée par la suite par les théories économiques sur la régulation qui influencera certains ornithologues, l'attention s'est focalisée sur l'agressivité comme comportement territorial, dans son effet aversif, et en tant que motif psychologique de constitution du territoire (qui expliquerait combats et parades), et de régulation de la surpopulation :

Les mots utilisés par certains ornithologues pour décrire les comportements sont éloquents, voire guerriers ou militaires : conflits, combats, challenges, contestations, attaques, poursuites, patrouilles, défense territoriale, quartier général (très fréquent pour signaler le centre du territoire à partir duquel l'oiseau chante), peinture de guerre (pour désigner les couleurs des oiseaux territoriaux). 
Ainsi le père de l'éthologie, Konrad Lorenz, publie un ouvrage au titre éloquent : L'Agression, une histoire naturelle du mal (1963). Cependant, derrière cette apparente univocité, un examen minutieux des observations ornithologiques met en lumière de multiples façons de faire territoire pour les oiseaux qui conduisent très tôt les scientifiques à se détacher de cette idée selon laquelle le territoire serait une «propriété ». Les choses s'avèrent bien plus complexes : les frontières des territoires, par exemple, n'auraient pas pour seule fonction de décourager les intrus. Vinciane Despret se fait ainsi l'écho de plusieurs dissonances, surtout d'intuitions pionnières qui mettent à l'épreuve et nuancent une perception trop humaine du territoire. C'est le cas notamment de Margaret Nice, ornithologue amatrice, qui invente dans les années 1920 un dispositif d'observation (le bagage individuel) pour les bruants chanteurs en Ohio, soucieuse de reconstituer leurs histoires de vie pour mieux comprendre ce qui importe pour eux au moment où ils construisent leurs territoires. D'autres, comme Warder Clyde Allee dans les années 1940 s'intéresseront davantage au problème de la sous-population (occulté jusqu'alors) et à l'idée selon laquelle il existerait des « communautés de vie » sous forme d' « assemblages écologiques », en d'autres termes des liens d'interdépendance entres les êtres d'un même territoire pour pallier à ce problème (mais cette idée à l'époque ne sera pas suivie). On s'aperçoit également que les mâles ne sont pas les seuls à chanter : les femelles chantent également. De même, en chantant, les mâles s'adressent avant tout aux autres mâles, ébranlant ainsi « la théorie de la sélection sexuelle », selon laquelle le chant aurait pour fonction d'attirer des femelles. Enfin, l'agressivité y apparaît davantage comme un jeu, un costume d'apparat : preuve en est que la proportion des oiseaux blessés dans les combats est infime et que la qualité du chant semble être un facteur déterminant dans le départ des intrus. Vinciane Despret considère Charles Moffat (dès 1903) comme précurseur de « la théorie des signaux honnêtes » : «L'oiseau affirme sa valeur et l'affirme de manière fiable, puisqu'il ne peut pas tricher, le chant, signal honnête, révélant une longue pratique » (p. 60). Le chant aurait selon Charles Moffat une fonction d'《 autoprésentation » : l'expérience chantée dissuadant l'intrus à poursuivre le combat et limitant ainsi les conflits. Cette découverte décisive incitera de plus en plus de chercheurs à s'intéresser à la question des apparences, à la force et à la singularité des chants. Le virage qui s'amorce est important : le territoire est de plus en plus considéré comme un lieu d'expressivité, un objet de performances. « L'agressivité n'est plus le motif au sens psychologique, ou la cause, de l'activité territoriale, elle en est le motif au sens esthétique ou musical, elle lui donne son style, sa forme de présentation, sa chorégraphie et ses gestes : l'agression devient de l'ordre du simulacre. » (p. 62) 
Le deuxième accord (composé des trois derniers chapitres « Possessions », «Agression », « Partitions polyphoniques ») de cet ouvrage poursuit les intuitions de Moffat et Allee et renforce cette idée selon laquelle le territoire serait moins une question spatiale qu'une question rythmique, en convoquant les philosophes Deleuze et Guattari, auteurs de Mille plateaux (et notamment le chapitre XI : «De la ritournelle ») qui ont pensé la question du territoire en termes de styles et de créativité, dans un double mouvement de déterritorialisation/ reterritorialisation. Plutôt que de territoires, Vinciane Despret préfère parler d'《 actes de territorialisation » : il s'agit d'insister sur le fait que le territoire est un acte qui affecte, territorialise «les milieux et les rythmes », générant des processus de métamorphoses chez les oiseaux. Chants, couleurs, parades seraient autant d'éléments d'un même masque que porteraient les oiseaux, devenant comme « possédés ». Le territoire constitué sur le mode de l'agressivité et via le chant relèverait alors du désir d'y résider. Croisant expériences ornithologiques et intuitions philosophiques, Vinciane Despret renforce ainsi l'hypothèse que « le territoire, c'est le lieu où tout devient rythme, paysage mélodique, motifs et contrepoints, matière à expression. Le territoire serait l'effet de l'art » (p. 110). Ce second accord est donc tout naturellement tourné vers le chant et la manière dont s'élabore un territoire sonore, opérant ainsi un retournement rhétorique en liant, contre toute attente, la question du territoire à celle du collectif et des « communautés de vie ». Elle pose par exemple l'hypothèse s'appuyant notamment sur des descriptions littéraires de Maylis de Kerangal à propos des chardonnerets d'Alger - que non seulement l'espace chanté fait corps avec l'oiseau, mais aussi qu'il en serait en quelque sorte une « extension » corporelle traduisant l'image sonore d'un « soi étendu » (dans ce moment particulier de débordement). Le chant serait une manière de faire corps avec un lieu qui s'invente et que l'oiseau souhaite rendre « propre à soi ». Les femelles ne choisiraient donc pas seulement un partenaire, mais également une « composition » sonore qui dirait la qualité du territoire, faisant du chant une cartographie et une musicographie :

Et si le chant est devenu l'expression d'un lieu, sans doute reconnaîtra-t-elle, dans sa signature, la hauteur des arbres, la présence d'un voisinage, paisible ou agité - on le verra, cela peut importer - la rugosité des roches, la présence d'une source elle-même chantante, l'ombre du couvert, le goût de ses fruits ou des insectes sous les feuilles et peut-être même la manière dont le soleil s'y fraye un chemin dans les frondaisons. (p. 125)

Ouvrant toujours plus cette question du territoire, elle revient sur la question des frontières, en s'appuyant sur l'ornithologue anglais James Fisher 
qui rappelle l'importante dimension sociale que requiert l'activité territoriale : les combats seraient ainsi des «stimulations sociales », et les frontières, en réalité très poreuses, des manières de s'inventer des voisins. En d'autres termes, le territoire serait 《matière à expression socialisée » (p. 145). D’ailleurs, plusieurs ornithologues semblent avoir constaté que les oiseaux établissent des solidarités entre voisins, contre les prédateurs. Il existerait une véritable coordination entre les oiseaux, et peut-être même entre les oiseaux et d'autres espèces, perceptibles au niveau du chant notamment. Vinciane Despret relate ainsi l'expérience du compositeur et bio-acousticien Bernie Krause ${ }^{1}$ qui a constaté, en enregistrant de véritables concerts sonores incluant les oiseaux (mais pas que), qu'il existe sur un même lieu « un partage du temps de parole » : le territoire serait donc un lieu d'accordage des chants. Enfin, ces observations font écho à celles de la bio-acousticienne Rachele Malavisi et d'une spécialiste de l'écologie des sons, Almo Farina, dans la forêt du Latium, qui ont mis en évidence des chœurs interspécifiques. Ces « cosmopolitiques expressives » constituent pour Vinciane Despret une perspective de recherche fascinante à venir en lien avec la question des territoires des oiseaux, en tant que réalité écologique dans leur dimension communautaire.

Vinciane Despret parvient ainsi à faire de l'écriture des savoirs ornithologiques un art de la palabre aussi chahuté que le territoire d'un oiseau au Printemps, agencé comme « une partition polyphonique » (le titre de son dernier chapitre), qui emplit la tête du lecteur et bouscule - l'air de rien, avec prudence et curiosité toute certitude sur une notion aussi controversée et brûlante d'actualité que le « territoire », à coup de plumes, pourrait-on dire... en permettant ainsi au lecteur de s'ouvrir à d'autres mondes : «Il faut écouter les oiseaux. Non pas pour qu'ils nous servent de modèles, car ils ont mieux à faire, mais pour laisser leurs mondes s'additionner aux nôtres $^{2}$ ». Une façon de (re)découvrir « l'extraordinaire du banal $^{3} \gg$.

1. Son travail fut exposé à la Fondation Cartier du 2 juillet 2016 au 8 janvier 2017 sous le titre « Le Grand Orchestre des Animaux ».

2. Extrait d'un entretien de Vinciane Despret sur http://www.mouvement.net/teteatete/ entretiens/vinciane-despret (propos recueillis par Aïnhoa Jean-Calmettes).

3. L'expression est de Philippe Vion-Dury qui constatait récemment le succès des livres sur les animaux et la nature. Voir « Renouer avec le vivant », Hors-série, $n^{\circ} 9$ de la revue Socialter coordonné par Baptiste Morizot, décembre 2020/février 2021. 